\title{
Maintaining Your Focus in Focus Groups: Avoiding Common Mistakes
}

By: Denise Côté-Arsenault, Dianne Morrison-Beedy

\section{This is the accepted version of the following article:}

Côté-Arsenault, D., \& Morrison-Beedy, D. (2005). Maintaining your focus in focus groups: Avoiding common mistakes. Research in Nursing \& Health, 28(2), 172-179. doi:10.1002/nur.20063.,

which has been published in final form at http://dx.doi.org/10.1002/nur.20063.

***@ (C) Wiley-Liss, Inc. Reprinted with permission. No further reproduction is authorized without written permission from Wiley-Liss, Inc. This version of the document is not the version of record. Figures and/or pictures may be missing from this format of the document. $* * *$

\begin{abstract}
:
Focus groups are used frequently in nursing research as a method for collecting qualitative data. However, due to inadequate planning or execution, the results can be of poor quality or even worthless. A well-defined purpose, carefully planned environment, and well trained personnel are discussed as the cornerstones to focus group success. This article sets the stage with a description of a well conducted focus group, then provides examples and a critique of less-thaneffective groups. Focus groups can be powerful and effective when their use is appropriate to the study purpose, the environment is non-judgmental and welcoming, and the personnel are skilled.
\end{abstract}

Keywords: focus groups | research methodology

\section{Article:}

Scenario: You have just finished conducting a focus group for your research. All of your training and planning has clearly paid off: the participants quickly became comfortable in the room that you selected, the participants' children are being cared for nearby by competent staff, the first questions asked by the moderator were unobtrusive, easy and quick to answer, and seemed to put everyone at ease. The moderator was personable, knowledgeable, and professional but did not direct the discussion. She had stated the purpose of the group discussion clearly and succinctly for all. Soon the participants were sharing their own experiences and opinions. The moderator's respect for each person became the model so that each story was listened to, nods among the participants were visible, and yet they were eager to point out similarities and differences in their own situations.

During the debriefing session following the focus group, each member of the research team agreed that new and unique topics and issues came out in the discussion. Conversation that went astray was easily and comfortably brought back to the interview question at hand. The moderator 
had done a skillful job. Synergy, energy, and individuality co-existed and were fostered within the group environment. The team had extensive field notes in addition to two sets of audio-tapes of the conversation so that a complete transcript was possible. The research team was satisfied, and in fact somewhat surprised, at what they had learned from the participants within a 2 hour session. Insights were gained, and new perspectives were revealed.

Clearly, this had been a successful and positive focus group.

This scenario illustrates many of the critical aspects of focus groups as a data collection methodology in which "a carefully planned series of discussions designed to obtain perceptions of a defined area of interest in a permissive, nonthreatening environment' takes place (Krueger \& Casey, 2000, p. 5). Unfortunately, not all focus groups are as successful as this illustration because many of the critical aspects of planning are often overlooked or underestimated. The facilitation of self-disclosure among participants should be the driving principle in focus groups (Krueger \& Casey) and, thus, human interaction can be both the key for success and the root of failure in this methodology. The purpose of all focus groups should be to gain understanding of a particular group's perspectives and opinions. Focus groups are useful for topic explorations, survey question development, and phenomenon descriptors. They are not appropriate, however, for case studies, in-depth individual cameos, or in situations where anonymity is essential. The choice of focus groups should be made with these strengths and weaknesses in mind.

It has become clear to us that what is often missing from the planning and conduct of focus groups is understanding and capitalizing on the human components. When done well, researchers can glean a great deal of information from a small group of individuals sitting in a circle with back and- forth conversation on a subject that they all have in common. With guidance, the participants are encouraged to share, ponder, expand, and explain their own views on the topic under discussion. Additional details regarding the initial planning and conduct of focus groups, including the role of moderator as well as the analysis of data obtained from this method, have been addressed elsewhere (Côté-Arsenault \& Morrison-Beedy, 1999; Kidd \& Parshall, 2000; Morrison-Beedy, Côté-Arsenault, \& Feinstein, 2001). The purpose of this discussion is to identify common mistakes in the use of focus groups and to provide guidance for avoiding them. Exemplars developed from our experiences, both as principal investigators (PIs) and as consultants, for research involving diverse populations and problems, will be presented.

\section{HISTORY OF FOCUS GROUPS}

The history of focus groups provides insights into the origins of some difficulties inherent in their use. Focus groups were developed during World War II for the purpose of conducting

military research on morale and on propaganda (Morgan, 1997). During this same period of time, social scientists began to use focus groups for marketing research, and to assess people’s responses to radio broadcasts and consumer perspectives on products. Further use of focus groups in more formal research was delayed until qualitative methods became more prevalent in 
the 1970s, and the unique contribution of focus groups to research was recognized (Kidd \& Parshall, 2000). However, focus groups were not commonly used in more mainstream, academic studies until the 1980s (Krueger \& Casey, 2000). As a result, focus groups were not developed alongside other qualitative methodologies that are grounded in philosophy. Indeed, focus groups are not a methodology in and of themselves; they are simply one approach to collecting qualitative data.

The data gathered during focus groups are qualitative: conversation transcripts, field notes of interactions, body language, and other nonverbal that will not be captured via audio-tape. Participant responses are guided by open-ended questions offered by the moderator, but what participants choose to say is spontaneous. For these reasons, the data are considered qualitative, or naturalistic as compared to the predetermined, controlled choices characteristic of quantitative data. Unlike other qualitative methodologies, however, focus groups are not conducted in a naturalistic setting. The choice of topic, participants, or setting should be carefully determined, not spontaneous. Research principles common to other qualitative methods, such as bias, bracketing, trustworthiness, and sampling, should be carefully implemented in conjunction with focus groups, as appropriate (Morrison-Beedy et al., 2001). Analysis for major themes or categories, and comparing and contrasting responses within the data are also qualitative in nature. As is true for all qualitative data collection, analysis should begin during the focus group and continue throughout subsequent groups and debriefing sessions. Data analysis is completed during more formal analysis sessions and the writing phase of the study.

Social discourse, the interaction integral to focus groups, is complex and must be fostered through careful choreography. People must feel comfortable to speak their minds in order for meaningful self-disclosure to occur. It is the researcher's job to create an environment where the invited individuals feel comfortable, motivated, and guided to share their thoughts and opinions on the identified topic. This does not occur by chance. Serial focus groups are not recommended because group processes, such as roles, and positions of power, begin to emerge. There is the danger of "group think" that would jeopardize the research fidelity, and groups could become an intervention, thus altering the participants' viewpoints (Parsons \& Greenwood, 2000). Each aspect of planning focus groups contributes to the overall success of the endeavor, and therefore each aspect must be carefully considered. Lack of attention to any one area could lead to an ineffective and thus unsuccessful focus group. Similar to the control of extraneous variables required in quantitative methods, significant attention to the "control" of detail is required when planning focus groups in order to create and maintain an inviting forum for open discussion.

\section{THREE CORNERSTONES TO FOCUS GROUP SUCCESS}

Based on our experiences and the literature, we have identified three key components needed for the successful conduct of focus groups: (a) keep the purpose of the study as the driving force, (b) establish an environment conducive to interactions, and (c) create skilled personnel through 
supervised training. While each component needs to be considered individually, they are also interdependent and share a tension that must be carefully balanced to optimize focus group outcomes.

Study Purpose: The Driving Force

The purpose of the research study being conducted should be the focus and driving force that dictates every subsequent decision when focus group methods are employed. The study purpose should be clearly articulated. When making decisions about the planning, development, or conduct of the focus group, the question should be asked, "Will this help us fulfill the purpose of the study?" With a clear purpose in mind, whom and where to recruit will become evident. A clear purpose should serve as a guide for the moderator if conversation goes astray. It will also be invaluable in discerning when to ask for clarification from the participants on particular areas of concern.

It is common, and tempting, to begin planning by writing out a list of interview questions, but we do not recommend this step until the purpose has been clearly developed. Indeed, asking the right questions in the best order is very important to answering the research questions. When appropriate, the interview guide should be carefully composed and used consistently throughout all focus group sessions. The questions should be open-ended, non-judgmental, and proceed from broad to specific topics, and from less to more sensitive topics. Each question should be used either to put the participants at ease initially and thus gain entry into their experiences or to acquire in-depth perspectives on the research topic (Greenwood \& Parsons, 2000).Well written questions provide an invitation for participants to tell details of their own stories and perspectives, and to share their experiences (Krueger \& Casey, 2000).

A debriefing session for the investigators, held immediately after participants leave, is an integral component of conducting each focus group session. This debriefing session helps the personnel reflect and refocus on the purpose of the study and progress made towards answering the research questions. Self-critique should be encouraged and changes made for the next session as appropriate. The first stages of analysis such as identification of major categories, initial themes, and general impressions should be shared and documented during these debriefing sessions.

Data collection is complete when an adequate number of groups have been conducted to gain answers to each research question, topics and opinions in subsequent groups are redundant, and the purpose of the study has been fulfilled.

\section{Environmental Design: Creating the Context}

The focus group environment consists of the participants, the setting and infrastructure, and the timing of group sessions. These variables contribute to the context within which everyone and everything comes together for each focus group. 
Participants are usually chosen by convenience, snowball, or purposive sampling. Group characteristics (age, gender, race, status, experience) must be carefully selected to provide the perspectives that are sought. Poor judgment used in deciding group composition can lead to participants who have their own agenda rather than being focused on the topic at hand. Groups should not be comprised of members with differing levels of power (e.g., supervisors and employees; care providers and patients). Such individuals should be divided into separate groups to prevent a sense of competition or disenfranchisement of some members. When power differentials exist, it is likely that those with less power will participate less fully or openly.

The appropriate group size is dependent on the topic, the gender, and the developmental levels of the participants. The PI must have adequate knowledge of the target population to determine group size and composition. However, it is commonly suggested to have groups of 6-10 (Krueger \& Casey, 2000; Morgan, 1997). Groups should be large enough to gain varying opinions and perspectives and small enough to allow each individual to participate fully and be heard. Group size can be problematic if the group is too small and each participant feels too much pressure to speak, or if the group is too large and members do not have ample opportunity to speak freely. The optimum combination of homogeneity of interest and common ground with adequate diversity of experiences is critical to productive interaction among members. Homogeneity on key variables is more important than general heterogeneity of the group (CôtéArsenault \& Morrison-Beedy, 1999; Dreachslin, 1998).

What is shared during focus groups is dependent on a setting that is comfortable, nonjudgmental, and safe so that participants are not inhibited in telling the group about what the researchers need to know. Self-disclosure, both positive and negative, must be possible and encouraged. The location should be accessible geographically and provide a private, nondistracting area where uninterrupted conversation can occur. The infrastructure of tables, chairs, temperature setting, and restrooms all contribute to a physically comfortable environment that will promote success.

Timing of focus groups refers to the time of day, day of the week, and possibly the period of time that has passed since the critical incident of interest. One approach to assessing appropriate timing for groups prior to conducting them is to ask some members of the targeted population for suggestions regarding meeting times. Gathering information about commonly shared schedules will assist in deciding ideal meeting times. It may also be helpful to ask participants what might prevent them from coming to a planned group so that these barriers can be addressed (e.g., child care needs, travel, and work schedules).

Research Personnel: Mix of Skill and Training

Creating and maintaining a successful focus group environment requires more than one research team member. Research team personnel for the focus group customarily include the PI, the moderator, and one or more note takers. Other specialized team personnel also may be needed. 
The PI has an oversight role throughout the study. The PI's familiarity with the population of interest and research topic combined with knowledge of the research process, are critical to the overall conceptualization and design of the study. This said, the importance of the role of the moderator cannot be overstated. Separating the PI and moderator roles is often suggested because this allows the moderator to focus on the task at hand and the PI to focus on evaluating the processes occurring within the group. Only a skilled moderator can provide the structure, stability, and freedom of expression that is required for successful discussions (Morrison-Beedy et al., 2001). Facilitating and accepting social interaction within certain guidelines, while always maintaining respect for each individual's contribution, are major components of this role. Development into the role of moderator, even for those who have moderated groups in the past, includes extensive training by the PI. The training would include modeling, rehearsal, and feedback about conducting groups with the specific population and also brainstorming difficulties, questions, or concerns that could arise. Ways to refocus and collect thoughts so that participants can be responded to in a sensitive manner should be practiced. An assistant moderator, or note taker, provides a second set of eyes and ears during the group discussion. This team member can take notes to capture on paper what cannot be audio-recorded, such as facial expressions and body language. This assistant also does necessary tasks required of successful groups, such as seeing to individual participant needs (e.g., restroom needs, water), insuring the recording of the sessions, and minimizing distractions.

\section{EXAMPLES OF PITFALLS AND COMMON MISTAKES}

We have compiled composite examples from flawed focus groups from our collective investigator and consultative experiences to demonstrate how lack of attention to purpose, environment, and/or personnel can derail the focus group process and impair the quality of the research data obtained using this method.

\section{Scenario One}

The PI was sitting in the empty room in the basement of a church, shaking her head, trying to figure out what had gone wrong. She had been so optimistic that focus groups would reveal key elements of minority women's experiences living with the chronic health condition they all had in common. A great deal of planning had been done. A minority nurse had been hired and trained to moderate the groups. Avenues of recruitment had been identified, and women had been contacted. The interview guide had been written carefully. The sessions seemed to begin well. Four to six women attended each session, there seemed to be group synergy, and the participants quickly seemed comfortable with each other. The moderator was welcoming and asked the planned opening questions. Then things went astray. The moderator became engaged with each of the women's stories, becoming more empathetic to their needs and experiences as they began discussing other health issues not targeted by the study. She answered the participants' questions about prescription medications, treatments, and other medical procedures. At the end of the focus group, the participants were pleased with their experience, they felt that their concerns and 
complaints were heard, their distress was acknowledged, and their health questions answered. In this focus group, symptom management and shared complaints took over the discussion, and the opportunity to address the purpose of the study - to understand their experiences with the disease in question-was missed.

Following the group, no debriefing sessions took place. The PI had been reassured by the moderator that things "had gone very well"' at the group. The PI did not listen to the tapes of the session before the next focus group was held. Ultimately little new knowledge had been gained about the challenges specific to the condition of concern, and the ultimate goal of the study was not achieved.

Discussion. The primary problem here was that the personnel were not doing their jobs. The moderator easily changed roles and became a caregiver instead of the moderator. The lack of focus led her to engage in reflective listening with the group participants. This was likely a seamless transition for her because of her nursing expertise. She was receiving positive feedback from the women around the discussion circle so she felt that she was meeting their needs; however, in doing so, she did not attend to the purpose of the study. No members of the research team provided the necessary evaluation that the discussion, however lively, was not answering the research question. Debriefing sessions could have put everyone back on track but they were not held, tapes were not reviewed between sessions, and thus the focus was not regained. If the PI had recognized that the focus had been lost, strategies to correct the direction of the group could have been implemented. For example, if the group was begun with a statement of the major goal of the group, as it should have been, this goal should be reiterated if the discussion goes astray. Cue cards with key reminders, such as a prepared phrase, " I hear what you are saying about_-, but what about_-' could be placed in front of the moderator, for ready assistance. Close supervision and possible retraining of the moderator between groups might be required. Unfortunately, the focus groups in this first scenario yielded little useful data, and the study questions were not answered.

\section{Scenario Two}

The research team was thrilled that everyone had come to the focus group to share their experiences of pregnancy subsequent to perinatal loss. Every chair was filled around the table, nine women plus the moderator, with a note taker who sat off to the side. Participants' children were being cared for nearby. Initially each woman told her story of pregnancy loss, and many heads nodded with empathy and understanding as group connections were made. As the subsequent questions were asked, most women responded easily and eagerly, leading to a lively discussion. In the final half hour, infant cries from across the hall became noticeable. This was distracting because it pulled the mothers' attention away from the discussion, and also because some of the participants did not yet have any live children of their own and found baby cries painful reminders of this fact. Discussion continued after one mother retrieved her child, but it never returned to the same flow of ideas that had preceded the disruption. 
At the debriefing session, the research team, including the moderator, identified two problems. First, one woman in the group never spoke again once the initial self-introductions were complete. There may have been several possible reasons for this woman's silence: she was a quiet person, there were too many voices louder than her own, she did not feel the need to speak because others were saying exactly what she would have said, or she may have disagreed with some of what she heard because she had different experiences but she could not find an opening into the discussion. Unfortunately, the reasons were never discerned. Clearly, the research team agreed, this was a problem, and because there had been so many participants the moderator had seen no easy solution. Everyone agreed that nine women were too many for a focus group on this sensitive topic. Changes were made in scheduling to prevent future large groups. Although it is common to overbook to deal with no shows or attrition, in this instance it was learned that scheduling only the optimum number of participants was best because, on this topic, fewer participants was better than too many. The second problem, the babysitting room set-up, was not so easily solved because there was no alternative room available, however, a different solution, hiring an additional babysitter, was agreed upon, and strategies for keeping the noise level down were put into place including having a stroller to take a noisy baby for a walk in the hall and toys were brought to keep children occupied.

Discussion. Despite the environmental challenges, a great deal was gained from this focus group session. But, the perspective of one participant was never gleaned, and it would have been prudent for the PI to follow-up with a phone call or individual interview with that woman, if possible. She could have been asked about her focus group experience as well as her own pregnancy after loss story. Although most sources on focus group methods recommend groups up to or exceeding nine participants, this group size was not effective with a sensitive and rarely discussed topic.

Providing babysitters for participants had been a good idea to enable women to attend but it also caused some disruption. More attention should have been paid to possible childcare issues and plans should have been in place for meeting the needs of the children. In this case the debriefing session provided a mechanism for discussion of the focus group process and aided in the resolution of both problems.

\section{Scenario Three}

HIV risk reduction was clearly an important topic. The PI knew the topic and population well but was at a loss about what went wrong with the focus group. The purpose of the study was to learn the sexual and substance use behaviors in men that relate to HIV risk and why they may or may not participate in HIV risk-reduction behaviors.

Half a dozen male participants were present for the session, along with the moderator and one additional team member - an appropriate size for such a focus group. The location was convenient, and the seating was comfortable for everyone. Drinks and finger foods were on the 
table in the center of the room. Participants were clearly interested in this important topic of HIV prevention and there was quiet chatter going on as people gathered. The moderator thanked the men for coming and explained the purpose of the study. After initial ice-breaking discussion, however, the conversation became rather non-productive. Questions such as, "Do men you know use condoms?" and "What do you know about condom use?” were asked as written from the highly structured interview guide, but the answers given were brief and non-disclosing. This awkward question and answer period was followed by a recitation of presumed HIV "facts" by several participants. It was not long before the men became restless. They began to argue with each other, in spite of the moderator's attempts to bring them back to the topic. Two people within the group started ridiculing another person, minimizing his contribution. Coming to his defense, another participant began to ask questions about possible myths about HIV risk, transmission, and prevention. Grateful for the diversion, and possibly being more comfortable in the role of health educator, the moderator recognized the knowledge deficits within the group and began to provide up-to-date information. The session ended calmly after $1 \frac{1}{2} 2$ hours, and the participants thanked the researchers for being able to participate.

Discussion. There were several difficulties in this scenario, but all of them could have been avoided with more careful preparation and insight. The two major flaws centered around the interview guide and the moderator In this instance, the choice of focus groups was appropriate and could have elicited rich data; however, the interview guide questions were directive and not open-ended. Written in a short answer format, they did not encourage exploration and expansion of diverse opinions or experiences, but rather, led to brief, dead-end answers. The moderator did not incorporate probes during the facilitation, and thus participants never had the back and forth conversation so important in focus groups. Ultimately they did not become engaged in the focus group process because they needed clearer guidance from the moderator.

The goals for the group were also in jeopardy because the moderator did not use prompts to elicit more information or reflection, nor did he control poor behavior among the participants. Interviewing skills needed to be used to draw out more personal responses from the men in the group. These skills should have been practiced during role playing sessions. Ground rules for the group, including the showing of respect to others and a welcoming of diverse opinions, should have been presented by the moderator at the opening of the session. With such ground rules, an environment with clear boundaries and a sense of safety would have been created. When behavior outside the boundaries was observed, the moderator could have quickly reminded the men of the group guidelines and their need to adhere to them. Because some participants were allowed to make negative personal remarks about others, the attempt to create a non-threatening environment was destroyed. Understandably, this failure made them self protective and led to non-productive talk in an atmosphere of mistrust. The general purpose of focus groups was not met because the participants did not feel comfortable sharing their thoughts and feelings.

Scenario Four 
Our final example took place within a nursing home setting. The researcher was trying to glean details about the routine morning care that residents received from the nursing staff. The PI served as her own moderator. Groups were scheduled at the end of the shifts during which the participants were on duty and took place in a conference room in the nursing home.

Several nurses’ aides, LPNs, and at least one RN attended each group. The planned questions seemed to work well to pull all of the participants into the discussion, but in all cases, despite stated ground rules which included that the purpose was not to critique anyone's care but to simply understand the normal order of events in their care, issues of power emerged. As more senior staff talked about the standard procedures and protocols, the nurses' aides became quiet. Apparently, they each had developed their own style of care, were more comfortable with some areas of personal care than others, and felt that their own judgment was adequate to choose which aspects of care should be given. The details of care actually provided became more and more vague as time progressed, and signs of fatigue became quite evident after 45-60 minutes. Therefore, the final questions yielded short answers, and participants became disinterested. The sessions were ended after 1 hour.

Debriefing sessions did not take place because the PI was alone in the focus groups. The sessions were tape recorded. After reviewing several tapes, the PI sought out our consultation.

Discussion. Group composition with their inherent power differentials caused some difficulty within these focus groups. Disparities in seniority impeded less senior participants from sharing their true feelings about their respective roles in patient care. Perhaps focus groups were not appropriate with the study participants described in this example. Alternatively, the aides could have been together in groups, and the professional staff, who were few in number, could have been interviewed individually. This strategy would have decreased, if not eliminated, the power differences that became a problem. While this example demonstrates status inequities, other power inequities can occur with other populations. For example, with pediatric and adolescent samples, age or developmental differences can produce the same negative effects.

The timing of the sessions, an issue of the environment, also likely contributed to the less than ideal results. It was assumed that the participants would prefer to come to groups immediately after work, but scheduling them at a time when participants had more energy might have increased the yield of richer data than did scheduling groups when participants were tired and wanted to go home.

\section{CONCLUSION}

The examples we have presented may seem like obvious mistakes that anyone could recognize and avoid, but they occur all too frequently. Without understanding or attending to the many issues regarding the study purpose, the environment created, and the personnel chosen and trained, researchers may not create the critical tension and balance needed between these three cornerstones. Our illustrations show the interrelations among each of these components. 
Clearly, the idea that focus groups are quick, easy, and ideal for novice researchers is a misconception. Successful focus groups require a great deal of attention to every detail, and the PI must have extensive knowledge not only about the population and topic but the methodology as well. However, when carefully planned and practiced by skilled researchers, focus groups can yield rich data that contribute a great deal to our nursing science.

\section{REFERENCES}

Côté-Arsenault, D., \& Morrison-Beedy, D. (1999). Practical advice for planning and conducting focus groups. Nursing Research, 48, 280-283.

Dreachslin, J.L. (1998). Conducting effective focus groups in the context of diversity: Theoretical underpinnings and practical implications, Qualitative Health Research, 8, 813-820.

Greenwood, J.,\& Parsons, M. (2000).Aguide to the use of focus groups in health care research: Part 2. Contemporary Nurse, 9, 181-191.

Kidd, P.S., \& Parshall, M.B. (2000). Getting the focus and the group: Enhancing analytical rigor in focus group research. Qualitative Health Research, 10, 293-308.

Krueger, R.A., \& Casey, M.A. (2000). Focus groups: A practical guide for applied research. Thousand Oaks, CA: Sage.

Morgan, D.L. (1997). Focus groups as qualitative research ( 2nd ed). Thousand Oaks, CA: Sage.

Morrison-Beedy, D., Côtô-Arsenault, D., \& Feinstein, N.F. (2001). Maximizing your results with focus groups: Moderator and analysis issues. Applied Nursing Research, 14, 48-53.

Parsons, M.,\&Greenwood, J. (2000).Aguide to the use of focus groups in health care research:

Part 1. Contemporary Nurse, 9, 169-180. 